\section{Diagnóstico da situação do aleitamento materno no Estado do Piauí, Brasil}

\author{
Diagnosis of the situation with breastfeeding in \\ Piauí State, Brazil
}

\author{
1 Secretaria Estadual de \\ Saúde do Piauí, Teresina, \\ Brasil. \\ 2 Instituto Fernandes \\ Figueira, Fundação Oswaldo \\ Cruz, Rio de Janeiro, Brasil. \\ 3 Universidade Federal do \\ Piauí, Teresina, Brasil. \\ ${ }^{4}$ Instituto de Saúde \\ Secretaria do Estado da \\ Saúde de São Paulo, São \\ Paulo, Brasil. \\ Correspondência \\ C. V. Ramos \\ Secretaria Estadual de Saúde \\ do Piauí. \\ Rua Padre Cícero Romão \\ Batista 450, Teresina, PI \\ 64016-285, Brasil. \\ nutricarmen2@yahoo.com.br
}

\begin{abstract}
This study aims to evaluate the situation with breastfeeding in the State of Piaui, Brazil, at the time of the 2006 vaccination campaign. The sample consisted of 1,963 infants $(<1$ year of age) from 45 municipalities. Medians and probabilities of breastfeeding categories were calculated by probit analysis. Multivariate logistic analysis was used to analyze the association between independent variables and partial and exclusive breastfeeding. The probabilities were: $16 \%$ for exclusive breastfeeding and $18 \%$ for predominant breastfeeding at 180 days of age and 58\% for breastfeeding at 361 days. Median duration was 67 days for exclusive breastfeeding and 200 days for breastfeeding. The breastfeeding rate was higher for children whose mothers belonged to social classes $C, D$, and $E(p=0.024)$ and lived in the countryside ( $p=0.009)$. Not nursing during the first 24 hours of life, using a pacifier, and bottle-feeding reduced the prevalence of breastfeeding $(p<0.001)$. The prevalence rates are below the recommended standards and reflect the need to prioritize actions to promote breastfeeding in the State.
\end{abstract}

Breast Feeding; Weaning; Infant Nutrition
Carmen Viana Ramos ${ }^{1,2}$ João Aprígio Guerra de Almeida 2 Norma Sueli Marques da Costa Alberto 1 João Batista Mendes Teles 3 Silvia Regina Dias Médici Saldiva ${ }^{4}$

\section{Introdução}

A promoção do aleitamento materno figura entre as intervenções viáveis, efetivas e de baixo custo que podem prevenir até $63 \%$ das mortes passíveis de ocorrer antes dos cinco anos de vida 1 . A prática de amamentar crianças exclusivamente por seis meses reduz o risco de infecções e previne déficits de crescimento ${ }^{2}$. Além disso, traz benefícios para a saúde da mulher, tais como: estimula a regressão uterina; auxilia no retorno ao peso inicial; previne o câncer de ovário, útero e mamas; diminui o risco de a mãe sofrer hemorragia e anemia no pós-parto 3 .

O relatório sobre a situação mundial da infância, publicado em 2006, revelou que 36\% das crianças menores de seis meses são aleitadas de forma exclusiva no mundo 4 . No Brasil, a última pesquisa de abrangência nacional foi realizada em 1999 e estimou uma prevalência de aleitamento materno exclusivo de $9,7 \%$ para a mesma faixa etária 5 . Estudos localizados realizados em cidades brasileiras, como Florianópolis (Santa Catarina) e João Pessoa (Paraíba) ${ }^{6}$, São Paulo 7 e São José do Rio Preto (São Paulo) ${ }^{8}$, Salvador 9 e Feira de Santana (Bahia) 10, além de no Distrito Federal 11, demonstram índices de aleitamento exclusivo aos seis meses inferiores a $18 \%$.

Estes resultados indicam que as taxas de aleitamento exclusivo alcançadas encontram-se aquém das recomendações oficiais preconizadas 12,13. Uma das possíveis explicações para es- 
sa constatação advém do fato de o aleitamento materno ser uma categoria híbrida, construída por atributos definidos pela natureza e cultura 14 , na qual variáveis como nível sócio-econômico, escolaridade, idade da mãe, trabalho materno e tipo de parto se associam de maneira distinta e sem um comportamento uniforme em relação à prática do aleitamento, a exemplo de relatos apresentados em inúmeras pesquisas 8,9,10,15,16,17,18.

$\mathrm{O}$ reconhecimento dessas questões sugere que mudanças devam ser operadas na prática assistencial de aleitamento materno, no intuito de melhorar os indicadores e tornar esse ato menos conflituoso para a mulher. Sob esse enfoque, iniciativas que privilegiem a rede de atenção primária, como um espaço onde a mulher possa ser vista na sua integralidade, configuram-se como estratégias eficientes para a promoção, proteção e apoio ao aleitamento materno 19 .

Por outro lado, os ajustes a serem operados na prática assistencial dependem de um conhecimento objetivo da realidade. Neste sentido, vale destacar que a última informação sobre aleitamento materno de âmbito estadual no Piauí foi a duração mediana do aleitamento materno, obtida em pesquisa realizada em 199120. O presente estudo se ergue na necessidade de conhecer a realidade atual, sendo, portanto, delineado com o objetivo de realizar um diagnóstico da situação do aleitamento materno no Estado do Piauí e apresentar os fatores a ele associados em crianças menores de um ano de idade. Os resultados servirão de subsídios para a formulação de políticas que visem a melhorar a prevalência do aleitamento materno no estado.

\section{Metodologia}

Em estudo transversal realizado durante a segunda etapa da campanha de vacinação em agosto de 2006 no Piauí, investigaram-se crianças com idade menor ou igual a 364 dias, presentes na campanha de multivacinação, acompanhadas das respectivas mães. Para tal, utilizou-se a estratégia de diagnósticos rápidos já validados em outros estudos 6,8,10,11. A meta estabelecida para a vacinação nessa faixa etária foi de 61.518 crianças para o estado. Com base nisso, foi dimensionada uma amostra representativa de 2.217 crianças, com um erro máximo de $2 \%$ e nível de $95 \%$ de confiança. Por motivos de perdas, como ausência da clientela nos postos no momento da pesquisa, recusa da mãe em responder o questionário ou preenchimento incorreto do instrumento por parte do entrevistador, a amostra final foi totalizada em 1.963 crianças, representando 11,46\% de não-resposta. Em relação às perdas,
Pereira 21 ressalta que não há unanimidade sobre os valores máximos permitidos e faz menção a limites que variam de $10 \%$ a $30 \%$. Para Babbie 22 , a taxa aceitável de resposta em pesquisas desse tipo é considerada muito boa quando atinge $70 \%$ ou mais. Neste estudo, em particular, foram atingidos $88,54 \%$ da amostra.

Para se chegar à unidade de análise última do estudo, foram consideradas três unidades amostrais: as microrregionais de saúde, o município e o posto de vacinação. A amostragem da pesquisa foi desenhada para incluir amostragem aleatória simples na escolha da regional de saúde. Dentro da regional selecionada, novamente uma amostragem casual simples para a seleção do município. Com base nesse critério, foram selecionados 45 municípios, dentre os 223 do estado, contemplando a zona rural e urbana. Os municípios selecionados são representativos do estado e encontram-se distribuídos uniformemente em oito regionais de saúde das 11 existentes.

O tamanho da amostra obedeceu ao quantitativo de crianças estabelecido pela meta de vacinação da Secretaria Estadual de Saúde do Piauí para cada um dos municípios. À exceção de Teresina, onde se trabalhou com $20 \%$ dos postos existentes, nos demais municípios todos os postos foram incluídos no estudo, contemplando proporcionalmente o número de crianças de cada região. A escolha da criança no posto ocorreu por meio de amostragem sistemática, cujo intervalo foi de três crianças.

A coleta de dados utilizou um questionário estruturado, aplicado por estudantes e profissionais de saúde dos municípios, previamente treinados pela coordenação da pesquisa. $\mathrm{O}$ questionário teve como base aquele aplicado na Pesquisa de Prevalência do Aleitamento Materno nas Capitais e Distrito Federal 5, sendo adaptado para o presente estudo. Dessa forma, decidiu-se pela supressão das questões relativas à vacinação e pela adição da variável classe econômica, a qual se baseou no critério Brasil usado pela Associação Brasileira de Empresas de Pesquisa (ABEP) 23. O instrumento era composto de 22 questões relativas a sexo da criança, idade, tipo de parto, alojamento conjunto, início do aleitamento - se foi nas primeiras 24 horas -, alimentação da criança nas últimas 24 horas, uso de mamadeira e chupeta, idade materna, escolaridade, trabalho materno, pré-natal (quando iniciou, quantas consultas, orientação para o aleitamento materno) e classe econômica. Vale ressaltar que não foram feitas perguntas recordatórias sobre a época do desmame e período de introdução de outros alimentos. Em relação ao aleitamento, obteve-se informação das últimas 24 horas. Foi realizada uma pesquisa-piloto na 
primeira etapa da Campanha de Vacinação, em junho de 2006, em quatro municípios e em condições idênticas às da pesquisa.

A classificação dos padrões de aleitamento considerada no estudo seguiu as recomendações da Organização Mundial da Saúde (OMS) 24, adotando-se as seguintes categorias de aleitamento: - Aleitamento materno exclusivo: a criança está recebendo somente leite materno, diretamente da mama ou extraído, e nenhum outro alimento líquido ou sólido, com exceção de gotas ou xaropes de vitaminas , minerais e/ou medicamentos; - Aleitamento materno predominante: a criança está recebendo além do leite materno, água ou bebidas à base de água, como sucos de frutas e chás;

- Aleitamento materno: a criança se alimenta com leite materno independente de consumo de outros líquidos ou alimentos sólidos/semisólidos, inclusive o leite não humano. Os questionários foram codificados e revisados para em seguida serem digitados e processados no programa SPSS, versão 13.0 (SPSS Inc., Chicago, Estados Unidos). As probabilidades de as crianças estarem em aleitamento materno exclusivo, aleitamento materno predominante e aleitamento materno nas diferentes idades foram estimadas por meio da análise de probitos, assim como a duração mediana do aleitamento materno exclusivo e aleitamento materno. A análise de probitos estima a proporção de crianças nas diferentes modalidades de aleitamento através de uma regressão linear ponderada de probitos (y) em relação às idades (x), considerando a amostra total das crianças em estudo 25. Dessa forma, os resultados são menos influenciados pelas flutuações amostrais que ocorreram mês a mês. Esta análise tem sido freqüentemente utilizada em estudos sobre aleitamento 8,11,26.

A relação entre a variável dependente aleitamento materno, em menores de um ano, expressa de forma dicotômica, e as variáveis independentes, para a mesma faixa de idade, foi analisada pela regressão logística múltipla, como forma de se reduzir a interferência dos fatores de confundimento. Os testes estatísticos para verificar a contribuição de cada variável no aleitamento materno através do modelo foram realizados com nível de significância de 0,05 . As variáveis independentes estudadas ficaram subdivididas da seguinte forma: tipo de parto (normal ou cesáreo); classe econômica (A, B ou C, D, E); anos de escolaridade (fundamental ou $>$ fundamental); número de consultas no pré-natal $(<6$ ou $6 \mathrm{e}+$ ); trabalho materno fora do lar (sim ou não); local de moradia (zona rural ou zona urbana); uso de chupeta (sim ou não); uso de mamadeira (sim ou não) e mamada nas primeiras 24 horas (sim ou não). As medidas de associação (odds ratio) foram estimadas por ponto e por intervalos de 95\% de confiança. O mesmo procedimento foi utilizado para estudar os fatores associados ao aleitamento materno exclusivo em menores de seis meses. Utilizou-se o programa SPSS, versão 13.0, para análise de probitos e da regressão logística.

Os gráficos correspondentes às estimativas das probabilidades das categorias de aleitamento materno, exclusivo e predominante, nas diversas idades, foram elaborados no aplicativo Excel para Windows (Microsoft Corp., Estados Unidos).

$\mathrm{O}$ projeto de pesquisa foi aprovado pelo Comitê de Ética em Pesquisa da Universidade Federal do Piauí, conforme prevê a Resolução $n^{o}$. 196/96 do Ministério da Saúde. A pesquisa foi financiada com recursos da Coordenação Geral da Política de Alimentação e Nutrição do Ministério da Saúde.

\section{Resultados}

O grupo estudado foi composto por 1.963 crianças menores de um ano, cujas variáveis sóciodemográficas podem ser observadas na Tabela 1. Destas, 80,23\% encontravam-se em aleitamento materno no dia da pesquisa. As probabilidades de as crianças menores de 365 dias receberem aleitamento materno são apresentadas na Figura 1 . As crianças têm $92 \%$ de probabilidade de receberem leite materno no primeiro mês de vida, e $58 \%$ no final do intervalo etário.

As probabilidades de as crianças estarem em aleitamento materno exclusivo e aleitamento materno predominante no curso de 180 dias podem ser observadas na Figura 2. As variações entre os extremos do intervalo etário foram de $72 \%$ para $16 \%$ e de $25 \%$ para $12 \%$ para o aleitamento materno exclusivo e aleitamento materno predominante, respectivamente. A duração da mediana do aleitamento materno foi de 200 dias; a do aleitamento materno exclusivo correspondeu a 67 dias. Cabe ressaltar que o percentual de crianças em aleitamento materno exclusivo na faixa de zero a 180 dias de vida foi de $41,45 \%$.

A Tabela 2 mostra os resultados da associação entre o aleitamento materno e os fatores relacionados à mulher e à criança. O aleitamento materno foi significativamente maior $(81 \%)$ entre aquelas mulheres que pertenciam às classes econômicas $\mathrm{C}$, D e E do que entre as pertencentes às classes econômicas A e B $(70,7 \%)(p=0,0024)$. Outro fator que mostrou associação significativa foi o local de moradia; morar na zona rural aumentou o percentual de aleitamento materno (88\%) em relação aos moradores da zona urbana 
Tabela 1

Distribuição percentual das crianças menores de um ano de acordo com as variáveis demográficas e sócio-ambientais. Piauí, Brasil, 2006.

\begin{tabular}{|c|c|c|}
\hline Variáveis & $\mathbf{n}$ & $\%$ \\
\hline \multicolumn{3}{|l|}{ Sexo da criança } \\
\hline Masculino & 999 & 50,9 \\
\hline Feminino & 964 & 49,1 \\
\hline \multicolumn{3}{|c|}{ Idade da criança (dias) } \\
\hline $0-30$ & 101 & 5,1 \\
\hline $31-60$ & 202 & 10,3 \\
\hline $61-90$ & 226 & 11,5 \\
\hline $91-120$ & 196 & 10,0 \\
\hline $121-150$ & 188 & 9,6 \\
\hline $151-180$ & 180 & 9,2 \\
\hline $181-270$ & 419 & 21,3 \\
\hline $271-364$ & 451 & 23,0 \\
\hline \multicolumn{3}{|l|}{ Idade materna (anos) } \\
\hline $12-19$ & 419 & 21,4 \\
\hline $20-24$ & 734 & 37,4 \\
\hline $25-35$ & 694 & 35,3 \\
\hline$>35$ & 106 & 5,4 \\
\hline Sem informação & 10 & 0,5 \\
\hline \multicolumn{3}{|l|}{ Local de moradia } \\
\hline Zona rural & 289 & 14,7 \\
\hline Zona urbana & 1.674 & 85,3 \\
\hline \multicolumn{3}{|l|}{ Freqüentou escola } \\
\hline Sim & 1.891 & 96,3 \\
\hline Não & 72 & 3,7 \\
\hline \multicolumn{3}{|l|}{ Anos de escolaridade } \\
\hline$<9$ & 1.042 & 55,0 \\
\hline $9 \mathrm{ou}+$ & 849 & 45,0 \\
\hline \multicolumn{3}{|l|}{ Trabalho materno } \\
\hline Sim & 418 & 21,3 \\
\hline Não & 1.545 & 78,7 \\
\hline \multicolumn{3}{|l|}{ Classe social } \\
\hline$A$ & 14 & 0,8 \\
\hline B & 126 & 6,4 \\
\hline $\mathrm{C}$ & 499 & 25,4 \\
\hline $\mathrm{D}$ & 835 & 42,5 \\
\hline$E$ & 489 & 24,9 \\
\hline
\end{tabular}

$(79 \%)(\mathrm{p}=0,009)$. As variáveis tipo de parto $(\mathrm{p}=$ $0,82)$, anos de escolaridade $(\mathrm{p}=0,13)$, número de consultas no pré-natal $(\mathrm{p}=0,85)$ e trabalho materno $(0,14)$ não se associaram de forma estatisticamente significante com o aleitamento materno (Tabela 2).

No que diz respeito às características das crianças, o hábito de não usar chupeta e mamadeira revelou uma forte associação estatística com o aleitamento materno. As crianças que não usavam chupeta apresentaram uma prevalência de aleitamento materno de $89 \%$; as que não usavam mamadeira, uma prevalência de $94 \%$, com uma chance significativamente maior de serem aleitadas $(p<0,001)$. Além desses fatores citados, a ocorrência da mamada nas primeiras 24 horas também se mostrou associada com a prática do aleitamento materno ( $\mathrm{p}<0,001)$. O aleitamento foi significativamente maior (82\%) entre as crianças que foram aleitadas no primeiro dia de vida em relação às que não foram (68\%) (Tabela 2).

Em termos dos fatores associados ao aleitamento materno exclusivo, o modelo de regressão logística revelou que a não-utilização da mamadeira amplia as chances de o aleitamento exclusivo ocorrer durante os seis primeiros meses de vida $(\mathrm{p}<0,001)$. As crianças que não usavam mamadeira tiveram uma prevalência de aleitamento exclusivo de $68,4 \%$, ao passo que as que a utilizavam, apenas $5,2 \%$. As outras variáveis estudadas não apresentaram associação estatisticamente significante com a ocorrência do aleitamento materno exclusivo ( $p>0,05)$.

\section{Discussão}

Os resultados encontrados no presente estudo possibilitam um diagnóstico da situação do aleitamento materno no Estado do Piauí, contribuindo para o planejamento de ações e medidas de intervenção na área. Para contextualização dos resultados aqui obtidos, foram utilizados os dois últimos estudos populacionais realizados em duas regiões circunscritas do estado: em Teresina, em 1999 5, no semi-árido, em 200527.

A análise dos dados obtidos nesta pesquisa, em relação aos verificados para Teresina 5, revelou maiores probabilidades de aleitamento materno exclusivo em todas as idades aqui apresentadas, com uma variação de $2,9 \%$ maior na idade de 30 dias e de $35,6 \%$ maior na idade de 180 dias. Em relação à categoria aleitamento materno, os resultados encontrados foram menores em todas as idades, com variação de $1,4 \%$ menor na idade de 30 dias e $0,7 \%$ menor aos 361 dias ${ }^{5}$.

A comparação dos resultados do presente estudo com o desempenho das outras capitais brasileiras, em 19995 , revelou que a probabilidade de $58 \%$ do aleitamento materno no final do primeiro ano de vida, no Piauí, foi maior que os resultados encontrados para a maioria das capitais, com exceção de Belém, no Pará (66,3\%) e Macapá, no Amapá $(64,1 \%)$. Quanto ao aleitamento materno exclusivo aos 180 dias de vida, a probabilidade de $16 \%$ encontrada nessa pesquisa também foi maior que na maioria das capitais, exceto para Belém (19,8\%), São Luís, no Maranhão $(18,1 \%)$ e Florianópolis, em Santa Catarina (18,4\%) 5. Vale 
Probabilidade do aleitamento materno * em crianças menores de um ano, segundo o modelo do probito. Piauí, Brasil, 2006.

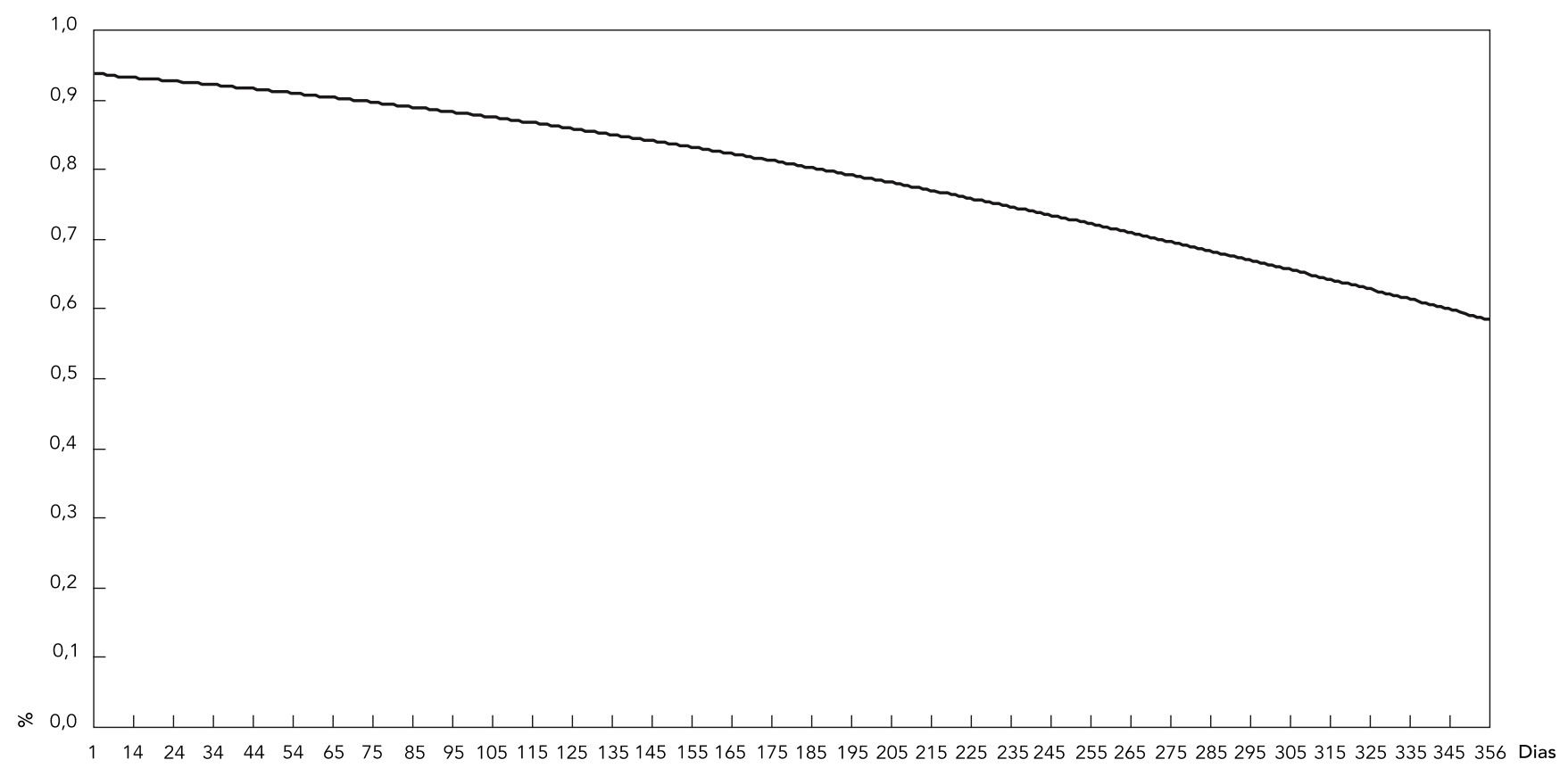

* Leite materno, independente do consumo de outros líquidos ou alimentos sólidos/semi-sólidos, inclusive o leite não humano.

salientar que a duração mediana de aleitamento materno exclusivo (67 dias) mostrou-se maior que a encontrada em todas as capitais em 1999, cujo maior valor foi de 63,6 dias para a cidade de Fortaleza, no Ceará 5 .

A probabilidade de aleitamento materno exclusivo na idade de 180 dias foi $38 \%$ menor que o obtido para o semi-árido piauiense em 200527. Em relação ao aleitamento materno, o resultado revelado para a idade de 361 dias foi $18,6 \%$ maior que o encontrado nessa região.

No que se refere ao aleitamento materno exclusivo, a probabilidade de $16 \%$ revelada neste estudo, ao final de 180 dias, também se apresentou maior que os valores relatados em pesquisas isoladas realizadas em Botucatu, São Paulo 26; Florianópolis e João Pessoa 6; Distrito Federal 11; São José do Rio Preto 8 e Salvador ${ }^{9}$, com exceção de Feira de Santana 10, onde a freqüência percentual nesta idade foi de $17,7 \%$. A duração mediana do aleitamento materno exclusivo foi maior que em todas essas cidades. No que tange ao aleitamento materno, na idade de 361 dias, o resultado se revelou maior que em todas as cidades supracitadas, sendo encontrada em Feira de Santana a maior freqüência de aleitamento materno (48,6\%) 10

Os maiores índices de aleitamento materno obtidos no presente trabalho, em relação às pesquisas citadas, devem ser considerados com cautela. Tal consideração se fundamenta na impossibilidade de realização de uma análise de tendências do aleitamento materno no Piauí e no fato de os resultados encontrados se mostrarem aquém das recomendações oficiais feitas pelo Ministério da Saúde 12 e pela OMS 13 .

A análise dos fatores relacionados à prevalência do aleitamento materno revelou que não mamar nas primeiras 24 horas, assim como utilizar chupeta e mamadeira, reduz as taxas de prevalência de aleitamento materno. Esses achados corroboram os estudos de Vieira et al. 10 no que diz respeito à associação com a mamada nas primeiras 24 horas e o de Figueiredo et al. 8, em relação ao uso da mamadeira como fator de redução no tempo de aleitamento materno. A utilização de chupeta imprimiu um risco 3,16 vezes maior para a interrupção do aleitamento materno em três municípios do Alto de Jequitinhonha, em Minas Gerais 18, e 5,99 vezes maior num estudo 
Probabilidade de aleitamento materno exclusivo * e aleitamento materno predominante ** em crianças com idade até 180 dias, segundo o modelo do probito. Piauí, Brasil, 2006

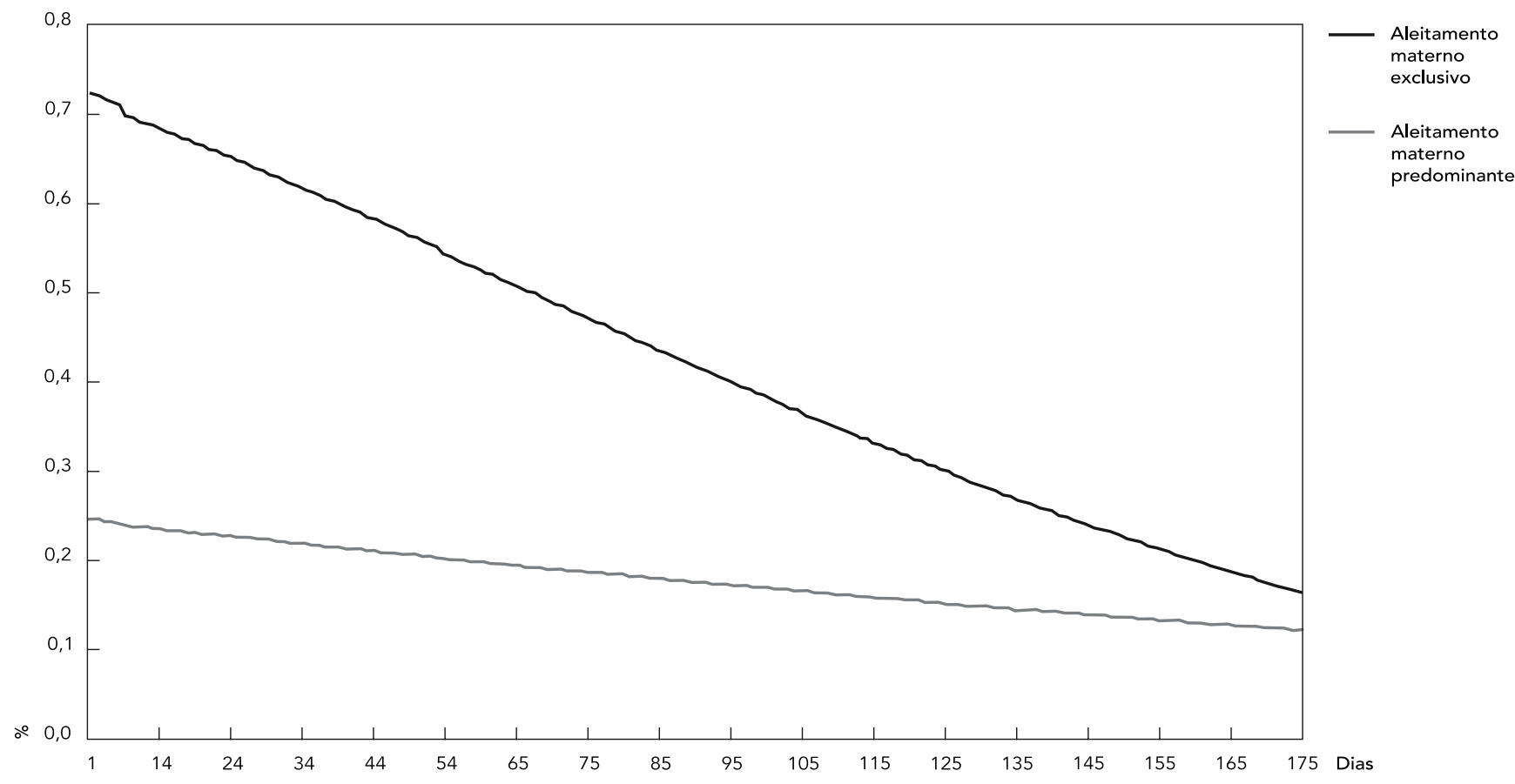

* Somente leite materno, sem nenhum outro alimento líquido ou sólido:

** Leite materno acrescido de água ou bebidas à base de água, como suco de frutas ou chás.

realizado em 111 municípios do Estado de São Paulo 28. Segundo a OMS 29, o uso de chupeta e mamadeira apresenta risco de transmissão de infecções, redução no tempo gasto na sucção do peito e interferência no aleitamento sob livre demanda, além de alterar a dinâmica oral.

Apesar do esforço das instituições governamentais para diminuir a utilização de chupetas e mamadeiras, as pesquisas revelam a persistência da adoção desses utensílios 8,10,28,30. No Piauí, a despeito de todo o investimento na capacitação profissional e em campanhas educativas, a análise dos dados deste estudo não revelou diferenças no hábito de utilização de chupetas e mamadeiras em relação ao verificado em Teresina em 1999 5. Essa constatação sugere a necessidade de estudos que possibilitem a análise de forma compreensiva dos determinantes culturais de tal prática.

O estudo de associação dos fatores inerentes à mulher com a prática do aleitamento evidenciou que o fato de pertencer às classes econômicas mais baixas $(\mathrm{C}, \mathrm{D}, \mathrm{E})$ e morar na zona rural aumentou a prevalência do aleitamento materno, enquanto as mulheres de classes econômicas mais altas (A e B) apresentam menores prevalências de aleitamento. Alguns trabalhos têm demonstrado que o baixo nível sócio-econômico, em especial a renda familiar, reduz as taxas de aleitamento 9,16,31, divergindo, portanto, do encontrado nesta pesquisa. A OMS 32 relaciona o aumento da freqüência de aleitamento materno ao padrão de desenvolvimento das sociedades, a qual apresenta fases de declínio e incremento da prevalência de aleitamento em diferentes momentos. Por esse raciocínio, as mulheres de melhor renda e mais escolaridade seriam as primeiras a valorizar o aleitamento, seguidas das que residem na zona rural com níveis sócio-econômicos mais baixos.

O fato de o estudo ter identificado uma maior duração de aleitamento materno nas 
Estimativas de odds ratio (OR) e intervalo de 95\% de confiança (IC95\%) da associação entre aleitamento materno e fatores das mães e das crianças. Piauí, Brasil, 2006.

\begin{tabular}{|c|c|c|c|c|c|c|c|}
\hline \multirow[t]{3}{*}{ Variáveis } & \multicolumn{4}{|c|}{ Aleitamento materno * } & \multirow[t]{3}{*}{ OR ** } & \multirow[t]{3}{*}{ IC95\% } & \multirow[t]{3}{*}{$p^{* \star *}$} \\
\hline & \multicolumn{2}{|c|}{ Sim } & \multicolumn{2}{|c|}{ Não } & & & \\
\hline & $\mathbf{n}$ & $\%$ & $\mathbf{n}$ & $\%$ & & & \\
\hline \multicolumn{8}{|l|}{ Tipo de parto } \\
\hline Normal & 957 & 81,0 & 219 & 19,0 & 1,0 & $0,7-1,3$ & 0,82 \\
\hline Cesáreo & 618 & 78,5 & 169 & 21,5 & & & \\
\hline \multicolumn{8}{|l|}{ Classe econômica } \\
\hline$A$ e $B$ & 99 & 70,7 & 41 & 29,3 & 0,6 & $0,4-0,9$ & 0,04 \\
\hline$C, D$ e $E$ & 1.476 & 81,0 & 347 & 19,0 & & & \\
\hline \multicolumn{8}{|c|}{ Anos de escolaridade } \\
\hline Fundamental & 844 & 81,0 & 198 & 19,0 & 1,2 & $1,0-1,7$ & 0,13 \\
\hline > Fundamental & 677 & 80,0 & 172 & 20,0 & & & \\
\hline \multicolumn{8}{|l|}{ Consultas pré-natal } \\
\hline$<6$ & 347 & 80,0 & 88 & 20,0 & 0,9 & $0,7-1,4$ & 0,85 \\
\hline 6 ou + & 1.216 & 80,5 & 294 & 19,5 & & & \\
\hline \multicolumn{8}{|l|}{ Trabalho materno } \\
\hline Não & 1.262 & 81,7 & 283 & 18,3 & 1,5 & $1,1-2,0$ & 0,14 \\
\hline Sim & 313 & 75,0 & 103 & 25,0 & & & \\
\hline \multicolumn{8}{|l|}{ Local de moradia } \\
\hline Zona urbana & 1.321 & 79,0 & 353 & 21,0 & 2,0 & $1,3-3,0$ & 0,01 \\
\hline Zona rural & 254 & 88,0 & 35 & 12,0 & & & \\
\hline \multicolumn{8}{|l|}{ Uso de chupeta } \\
\hline Não & 1.098 & 89,0 & 140 & 11,0 & 2,8 & $2,2-3,7$ & 0,000 \\
\hline Sim & 477 & 66,0 & 248 & 34,0 & & & \\
\hline \multicolumn{8}{|l|}{ Uso de mamadeira } \\
\hline Não & 919 & 94,0 & 54 & 6,0 & 6,4 & $4,8-8,9$ & 0,000 \\
\hline Sim & 652 & 66,0 & 333 & 34,0 & & & \\
\hline \multicolumn{8}{|c|}{ Mamada após 24 horas do parto } \\
\hline Não & 158 & 68,0 & 74 & 32,0 & 1,7 & $1,2-2,4$ & 0,000 \\
\hline Sim & 1.415 & 82,0 & 314 & 18,0 & & & \\
\hline
\end{tabular}

* Leite materno, independente do consumo de outros líquidos ou alimentos sólidos/semi-sólidos, inclusive o leite não

humano;

** Resultado da regressão logística.

classes menos favorecidas sugere uma melhor difusão de saberes e práticas nesse estrato social, à semelhança do estudo conduzido em Feira de Santana 10.

Faleiros et al. 31, analisando os fatores que influenciam a decisão e duração do aleitamento materno, alertam sobre a necessidade de se estudarem, além dos fatores comumente pesquisados, outras causas para o desmame precoce, como as relacionadas ao ambiente, à personalidade materna, às suas emoções, às relações familiares, às influências culturais e às respostas das mulheres aos diferentes problemas do cotidiano, incluindo o papel dos meios de comunicação e indústria de alimentos infantis. Assim sendo, as variáveis contextuais assumem um papel fundamental. Em adição, a mulher precisa se sentir apoiada para a realização do ato de amamentar, o que já foi bastante evidenciado em estudos que privilegiam a análise compreensiva da prática do aleitamento 33,34,35,36,37. Em desenhos de futuros estudos, vale atentar para a necessidade de coletar dados que permitam realizar a análise de fatores associados à prática de aleitamento materno não contemplados nesta pesquisa.

A determinação do perfil atual de aleitamento materno no Estado do Piauí e os fatores associados à sua duração representam a principal contribuição deste estudo. Mais do que um re- 
trato momentâneo da situação do aleitamento no estado, as taxas ora determinadas se configuram em demarcadores para futuros estudos e análise de tendências.

Apesar dos maiores índices obtidos, especialmente em relação ao aleitamento exclusivo, os resultados encontram-se ainda muito aquém das recomendações oficiais. Nessa perspectiva, o investimento em ações estratégicas que produzam transformações em favor do aleitamento materno deve ser considerado como prioritário pela política de saúde no estado.

\section{Resumo}

O objetivo do estudo foi realizar um diagnóstico da situação do aleitamento materno no Piauí, Brasil, durante a campanha de vacinação de 2006. A amostra constituiu-se de 1.963 crianças menores de um ano em 45 municipios. As medianas e probabilidades das categorias de aleitamento foram realizadas pela análise de probitos. Para análise da associação entre as variáveis do estudo e aleitamento materno/aleitamento materno exclusivo, utilizou-se o modelo de regressão logística multivariada. As probabilidades encontradas foram: 16\% para o aleitamento materno exclusivo, $18 \%$ para o predominante na idade de 180 dias e 58\% para aleitamento materno aos 361 dias. A duração mediana foi de 67 dias para o aleitamento materno exclusivo e 200 para aleitamento materno. Entre as crianças cujas mães pertenciam às classes econômicas $C, D$ e $E$ ( $p=$ $0,024)$ e moravam na zona rural $(p=0,009)$, o aleitamento materno foi maior. Não mamar nas primeiras 24 horas e utilizar chupeta e mamadeira reduziram a prevalência do aleitamento materno $(p<0,001)$. As prevalências encontram-se aquém das recomendações e refletem a necessidade de priorização das ações voltadas para o aleitamento no estado.

Aleitamento Materno; Desmame; Nutrição do Lactente

\section{Colaboradores}

C.V. Ramos elaborou a estrutura do artigo, detalhamento da análise, gráficos e tabelas, e revisou após pareceres. J. A. G. Almeida realizou a revisão final do artigo e revisou após os pareceres. N. S. M. C. Alberto supervisionou a coleta de dados, discutiu os resultados. J. B. M. Teles foi responsável pela amostragem, tratamento estatístico do projeto, discutiu os resultados, revisou a metodologia do artigo. S. R. D. M. Saldiva contribuiu na análise final dos dados, discutiu os resultados, fez a revisão da metodologia.

\section{Agradecimentos}

Cassandra Sena, Juzilene Lopes e Deusilene Brito, Técnicas da Supervisão de Ações Básicas de Saúde e Alimentação e Nutrição da Coordenação de Ações da Saúde da Criança e Adolescente, Secretaria Estadual de Saúde do Piauí (SESAPI). Vilma Brito, Supervisora do Aleitamento Materno da SESAPI. Danielle Monteiro, Técnica em Informática. Raimunda Damasceno, Coordenadora da Imunização da SESAPI. Franklin Borges, Professor de Inglês. 


\section{Referências}

1. Jones G, Steketee RW, Black RE, Bhutta ZA, Morris SS. How many child deaths can we prevent this year? Lancet 2003; 362:65-71.

2. World Health Organization. Family and community practices that promote child survival, growth and development: a review of the evidence. Geneva: World Health Organization; 2004.

3. Organização Pan-Americana da Saúde. Semana mundial da amamentação 2006. http:/ / www.opas. org.br/mostrantp.cfm?codigodest=593 (acessado em 23/Mai/2007).

4. Fundo das Nações Unidas para a Infância. Situação mundial da infância 2006 - excluídas e invisíveis. New York: Fundo das Nações Unidas para a Infância; 2005.

5. Ministério da Saúde. Pesquisa de prevalência do aleitamento materno nas capitais e Distrito Federal. Brasília: Ministério da Saúde; 2001.

6. Kitoko PM, Réa MF, Venancio SI, Vasconcelos ACCP, Santos EKA, Monteiro CA. Situação do aleitamento materno em duas capitais brasileiras: uma análise comparada. Cad Saúde Pública 2000; 16:1111-9.

7. Rea MF, Venancio SI, Batista LE, Santos RG, Greiner T. Possibilidades e limitações da amamentação entre mulheres trabalhadoras formais. Rev Saúde Pública 1997; 31:149-56.

8. Figueiredo MO, Sartorelli DS, Zan TAB, Garcia E, Silva LC, Carvalho FLP, et al. Inquérito de avaliação rápida das práticas de alimentação infantil em São José do Rio Preto, São Paulo, Brasil. Cad Saúde Pública 2004; 20:172-9.

9. Oliveira LPM, Assis AM, Gomes GSS, Prado MS, Barreto ML. Duração do aleitamento materno, regime alimentar e fatores associados segundo condições de vida em Salvador, Bahia, Brasil. Cad Saúde Pública 2005; 21:1515-30.

10. Vieira GO, Almeida JAG, Silva LR, Cabral VA, Netto PVS. Fatores associados ao aleitamento e desmame em Feira de Santana, Bahia. Rev Bras Saúde Matern Infant 2004; 4:143-50.

11. Sena MCF, Silva EF, Pereira MG. Prevalência do aleitamento materno no Distrito Federal, Brasil. Cad Saúde Pública 2002; 18:613-21.

12. Ministério da Saúde/Organização Pan-Americana da Saúde. Guia alimentar para crianças menores de dois anos. Brasília: Ministério da Saúde/Organização Pan-Americana da Saúde; 2005.

13. World Health Organization. The optimal duration of exclusive breastfeeding. Geneva: World Health Organization; 2001. (Note for the Press, 7).

14. Almeida JAG, Novak FR. Breastfeeding: a natureculture hybrid. J Pediatr 2004; 80 Suppl 5:119-25.

15. Weinderpass E, Barros FC, Victora CG, Tomasi E, Halpern R. Incidência e duração da amamentação conforme tipo de parto: estudo longitudinal no Sul do Brasil. Rev Saúde Pública 1998; 32:225-31.

16. Kummer SC, Giugliani ERJ, Susin LO, Folletto JL, Lermen NR, Wu VYJ, et al. Evolução do padrão do aleitamento materno. Rev Saúde Pública 2000; 34:143-8.
17. Bueno MB, Souza JMP, Souza SB, Paz SMRS, Gimeno SGA, Siqueira AAF. Riscos associados ao processo de desmame entre crianças nascidas em hospitais universitários de São Paulo, entre 1998 e 1999: estudo de coorte prospectivo do primeiro ano de vida. Cad Saúde Pública 2003; 19:1453-60.

18. Silveira FJF, Lamounier JA. Fatores associados à duração do aleitamento materno em três municípios na região do Alto Jequitinhonha, Minas Gerais, Brasil. Cad Saúde Pública 2006; 22:69-77.

19. Oliveira MIN, Camacho LAB, Souza IEO. Promoção, proteção e apoio à amamentação do Rio de Janeiro, Brasil: uma política de saúde pública baseada em evidência. Cad Saúde Pública 2005; 21:1901-10.

20. Fundo das Nações Unidas para a Infância/ Universidade Federal do Piauí/Secretaria Estadual de Saúde do Piauí. Crianças e adolescentes no Piauí: saúde, educação e trabalho. Teresina: Fundo das Nações Unidas para a Infância; 1992.

21. Pereira MG. Epidemiologia: teoria e prática. Rio de Janeiro: Editora Guanabara Koogan; 1995.

22. Babbie E. Métodos de pesquisa de survey. Belo Horizonte: Editora UFMG; 1999.

23. Associação Brasileira de Empresas de Pesquisa. Critério de classificação econômica Brasil. http:// www.abep.org.br (acessado em 23/Abr/2007).

24. Division of Diarrhoeal and Acute Respiratory Disease Control, World Health Organization. Indicators for assessing breast-feeding practices. Geneva: World Health Organization; 1991.

25. Finney DJ. Probit analysis. 3rd Ed. Cambridge: Cambridge University Press; 1980.

26. Carvalhaes MABL, Parada CMGL, Manoel CM, Venâncio SI. Diagnóstico da situação do aleitamento materno em área urbana do Sudeste do Brasil: utilização de metodologia simplificada. Rev Saúde Pública 1998; 32:430-6.

27. Ministério do Desenvolvimento Social e Combate à Fome. Chamada nutricional: um estudo sobre a situação nutricional das crianças do semi-árido brasileiro. Brasília: Ministério do Desenvolvimento Social e Combate à Fome; 2006. (Caderno de Estudos Desenvolvimento Social em Debate).

28. Contrim LC, Venancio SI, Escuder MML. Uso de chupeta e amamentação em crianças menores de quatro meses no Estado de São Paulo. Rev Bras Saúde Matern Infant 2002; 2:245-52.

29. Organização Mundial da Saúde/Organização PanAmericana da Saúde. Evidências científicas dos dez passos para o sucesso do aleitamento materno. Brasília: Organização Mundial da Saúde/Organização Pan-Americana da Saúde; 2001.

30. Soares MAM, Giugliani ERJ, Braun ML, Salgado ACN, Oliveira AP, Aguiar PR. Uso da chupeta e sua relação com o desmame precoce em população de crianças nascidas em Hospital Amigo da Criança. J Pediatr (Rio J) 2003; 79:309-16.

31. Faleiros FTV, Trezza EMC, Carandina L. Aleitamento materno: fatores de influência na sua decisão e duração. Rev Nutr PUCCAMP 2006; 19:623-30.

32. World Health Organization. The World Health Organization's infant feeding recommendation. Bull World Health Organ 1995; 73:165-74. 
33. Nakano MS. O aleitamento no cotidiano feminino [Tese de Doutorado]. São Paulo: Escola de Enfermagem de Ribeirão Preto, Universidade de São Paulo; 1996.

34. Silva IA. Amamentar: uma questão de assumir riscos ou garantir benefícios. São Paulo: Editora Robe; 1997.

35. Silva IA. Construindo perspectivas sobre assistência em amamentação: um processo interacional [Tese de Livre Docência]. São Paulo: Escola de Enfermagem, Universidade de São Paulo; 1999.
36. Ramos CV, Almeida JAG. Alegações maternas para o desmame: estudo qualitativo. J Pediatr (Rio J) 2003; 79:385-90.

37. Souza MHN. A mulher que amamenta e suas relações sociais: uma perspectiva compreensiva de promoção e apoio [Tese de Doutorado]. Rio de Janeiro: Escola de Enfermagem Anna Nery, Universidade Federal do Rio de Janeiro; 2006.

Recebido em 03/Ago/2007

Versão final reapresentada em 17/Dez/2007

Aprovado em 02/Jan/2008 